\title{
O tratamento farmacológico do transtorno bipolar: uma revisão sistemática e crítica dos aspectos metodológicos dos estudos clínicos modernos
}

\section{The pharmacological treatment of bipolar disorder: a systematic and critical review of the methodological aspects of modern clinical trials}

\author{
Elie Cheniaux ${ }^{1,2}$ \\ ${ }^{1}$ Instituto de Psiquiatria, Universidade Federal do Rio de Janeiro (UFRJ), Rio de Janeiro, RJ, Brazil \\ ${ }^{2}$ Faculdade de Ciências Médicas, Universidade do Estado do Rio de Janeiro (UERJ), Rio de Janeiro, RJ, Brazil
}

Resumo

Objetivo: Revisar sistematicamente os principais estudos clínicos sobre o tratamento farmacológico do transtorno bipolar e fazer uma análise crítica de seus aspectos metodológicos. Método: Realizou-se uma busca nas bases de dados Medline, ISI e PsycINFO, utilizando-se os seguintes termos de busca: "bipolar", "randomized", "placebo" e "controlled". Foram selecionados estudos clínicos randomizados, duplo-cegos e controlados por placebo sobre o tratamento farmacológico do transtorno bipolar. Além disso, de acordo com os nossos critérios, as amostras deveriam ser de no mínimo 100 pacientes e a substância testada deveria ser usada como monoterapia. Resultados: 34 artigos se adequaram aos critérios de seleção. Todas as substâncias atualmente indicadas para mania, depressão bipolar e para o tratamento de manutenção foram mais eficazes que o placebo em pelo menos um estudo. Todavia, esses estudos tiveram amostras altamente selecionadas, altas taxas de abandono e baixas taxas de resposta clínica. Conclusão: Os modernos estudos clínicos sobre o tratamento farmacológico do transtorno bipolar apresentam algumas importantes limitaçôes metodológicas. Assim, seus resultados devem ser considerados com cautela.

Descritores: Transtorno bipolar; Farmacoterapia; Revisão; Metodologia; Ensaios clínicos como assunto
Abstract

Objective: To review systematically the main clinical trials on the pharmacological treatment of bipolar disorder and to make a critical analysis of their methodological aspects. Method: A search in Medline, ISI and PsycINFO databases was conducted, using the following search terms: "bipolar", "randomized", "placebo"e "controlled". Randomized, double-blind, placebo-controlled clinical trials on the pharmacological treatment of bipolar disorder were selected. Besides, according to our criteria, samples had to consist of at least 100 patients and experimental drug had to be used as monotherapy. Results: 34 articles met our selection criteria. All drugs currently indicated for mania, bipolar depression and maintenance treatment of bipolar disorder were more effective than placebo in at least one clinical trial. However, these studies had highly selected samples, high dropout rates and low response rates. Conclusion: Modern clinical trials on pharmacological treatment of bipolar disorder have important methodological limitations. So, their results should be taken with caution.

Descriptors: Bipolar disorder; Pharmacotherapy; Review; Methodology; Clinical trials as topic

\section{Introdução}

O tratamento de indivíduos com transtorno bipolar é uma tarefa altamente complexa. Antes de tudo, porque envolve estratégias distintas nas diferentes fases da doença: mania, depressão e eutimia. Até o momento, receberam aprovação do Food and Drug Administration (FDA), a agência americana responsável pelo controle de medicamentos e alimentos, para o tratamento da mania o lítio, a clorpromazina, a carbamazepina, o divalproato e diversos antipsicóticos atípicos - a risperidona, a olanzapina, a quetiapina, a ziprasidona, o aripiprazol ${ }^{1} \mathrm{e}$, mais recentemente, a asenapina ${ }^{2}$. Para a depressão bipolar, foram aprovados apenas a

E-mail: echeniaux@gmail.com 
combinação olanzapina-fluoxetina e a quetiapina ${ }^{3}$. Por fim, o FDA recomenda para o tratamento de manutenção o lítio, a olanzapina, o aripiprazol e a lamotrigina ${ }^{4}$. Todas essas substâncias, exceto a clorpromazina ${ }^{5}$, demonstraram sua eficácia por meio de estudos clínicos com grandes amostras, randomizados, duplo-cegos e controlados por placebo.

Diante dessa multiplicidade de opções terapêuticas consideradas eficazes, poderíamos imaginar um cenário bastante otimista para os pacientes que sofrem de transtorno bipolar. No entanto, o que se observa é que grande parte desses pacientes, mesmo seguindo um tratamento regular e adequado, apresenta uma evolução desfavorável. Em um estudo multicêntrico recente ${ }^{6}$, 1.469 bipolares, submetidos ao "melhor tratamento disponível", foram acompanhados durante dois anos. Neste período, apenas $58,4 \%$ dos pacientes apresentaram em algum momento remissão dos sintomas afetivos, e, entre aqueles que conseguiram ficar assintomáticos, $48,5 \%$ tiveram recorrências.

Em função desse aparente contraste entre a observação clínica diária e os resultados dos mais importantes estudos clínicos sobre a eficácia do tratamento farmacológico do transtorno bipolar, realizamos uma revisão sistemática desses estudos com o objetivo de fazer uma análise crítica de seus aspectos metodológicos.

\section{Método}

Foi realizada uma revisão sistemática da literatura científica sobre a eficácia do tratamento farmacológico do transtorno bipolar. Buscaram-se especificamente estudos clínicos com grandes amostras, randomizados, duplo-cegos e controlados por placebo. Foram utilizadas as bases de dados Medline, ISI e PsycINFO, no dia 5 de maio de 2010. Não houve nenhuma restrição quanto ao período da publicação. Os termos de busca empregados foram "bipolar", "randomized", "placebo" e "controlled".

Só foram selecionados estudos nos quais a substância testada foi empregada como monoterapia. A única exceção em relação a este critério foi a combinação olanzapina-fluoxetina, devido ao fato de esta associação de substâncias ter sido lançada no mercado como um único medicamento. Embora, na prática clínica diária, os pacientes que sofrem de transtorno bipolar sejam muito frequentemente tratados com mais de um medicamento ao mesmo tempo, o FDA, via de regra, analisa a eficácia de uma determinada substância com base em estudos que a empregam como monoterapia.

Com o objetivo de eliminar estudos com amostras muito pequenas, estipulou-se que só seriam incluídos aqueles com pelo menos 100 pacientes. Além disso, as amostras deveriam ser constituídas apenas por indivíduos adultos.

Não houve procura por estudos não publicados. Quando resultados diferentes relativos a uma mesma amostra eram publicados em artigos diferentes, somente o estudo original foi selecionado. Assim, estudos com análises ad hoc de subamostras ou que representavam prolongamentos de estudos originais não foram considerados.

\section{Resultados}

$\mathrm{Na}$ busca inicial, encontrou-se um total de 647 referências no Medline, 562 no ISI e 235 no PsycINFO. Após análise dos resumos e, em alguns casos, do texto integral do artigo, foram selecionados 34 artigos. A maioria das referências excluídas consistiu em artigos de revisão, relatos de caso e cartas para o editor. Entre os estudos clínicos não selecionados, foram comuns os abertos ou não comparativos, com duas ou mais substâncias combinadas, ou com amostras formadas exclusivamente por crianças ou adolescentes.

\section{Tratamento da mania bipolar}

A Tabela 1 apresenta as informaçôes dos 21 artigos encontrados sobre o tratamento farmacológico da mania que se adequaram aos critérios de seleção. Os 21 artigos se referem a 24 estudos clínicos, já que um dos artigos ${ }^{7}$ apresentou os resultados de quatro estudos clínicos. Nesses estudos, 11 diferentes substâncias foram comparadas com placebo, sendo que em dez deles havia mais de uma substância ativa sendo testada. Em todos os estudos, foi utilizada a redução no escore total de uma escala de sintomas maníacos como medida primária de eficácia. Apenas três estudos ${ }^{8-10}$ não permitiram a inclusão de pacientes em estado misto nas amostras.

De acordo com os resultados, observou-se superioridade do lítio sobre o placebo em cinco estudos, publicados em quatro $\operatorname{artigos}^{7,8,11,12}$, da carbamazepina em dois estudos ${ }^{13,14}$, do divalproato em dois estudos ${ }^{11,15}$, da olanzapina em cinco estudos ${ }^{16-20}$, do aripiprazol em quatro estudos ${ }^{12,21-23}$, da ziprasidona em dois estudos $^{24,25}$, da risperidona em dois estudos ${ }^{9,26}$, da quetiapina em dois estudos ${ }^{8,10}$, da asenapina em dois estudos ${ }^{16,17}$ e do haloperidol em dois estudos ${ }^{10,23}$.

Todos os quatro estudos com o topiramato foram negativos ${ }^{7}$. Em dois deles, o topiramato também foi inferior ao lítio. Em dois estudos, o divalproato foi equivalente ao placebo ${ }^{20,27}$. Em um desses dois estudo ${ }^{20}$, o divalproato também não se distinguiu da olanzapina, embora esta tenha apresentado um resultado positivo. Num estudo no qual tanto o haloperidol como a quetiapina foram superiores ao placebo, o haloperidol também foi superior à quetiapina ${ }^{10}$. Por fim, num estudo em que a olanzapina e a asenapina foram consideradas eficazes, a olanzapina levou a uma redução significativamente maior na escala de mania do que a asenapina ${ }^{17}$.

Apenas em nove estudos há informações sobre a taxa de virada para depressão durante o tratamento. Com relação a esse aspecto, o lítio ${ }^{7,8,12}$ o topiramato ${ }^{7}$ o aripiprazol ${ }^{12,23}$, a olanzapina ${ }^{16-19}$, a quetiapina $^{8,10}$, o haloperidol ${ }^{10,23}$ e a asenapina ${ }^{16,17}$ não se distinguiram do placebo.

Nos estudos sobre o tratamento da mania, os tamanhos das amostras foram bastante elevados, variando entre $115^{19}$ e $521^{20}$ pacientes. Todavia, uma parte significativa dos pacientes avaliados na triagem inicial não foi incluída no estudo. Foram comuns entre os critérios de exclusão um risco importante de violência ou suicídio e comorbidade com um transtorno de abuso ou dependência de substância ou com doença clínica. Dos 24 estudos, 

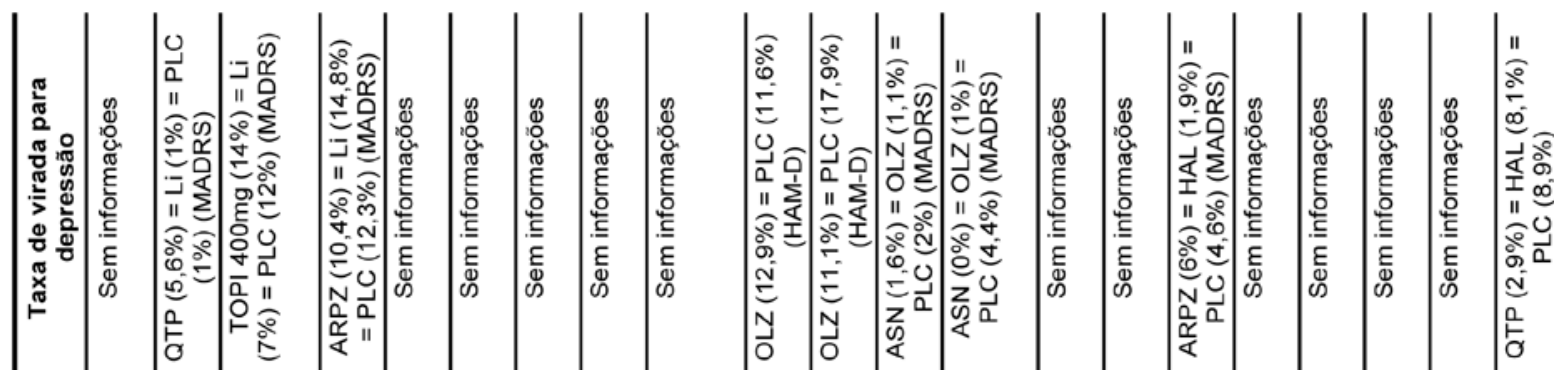

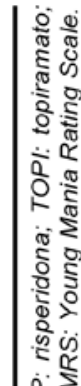

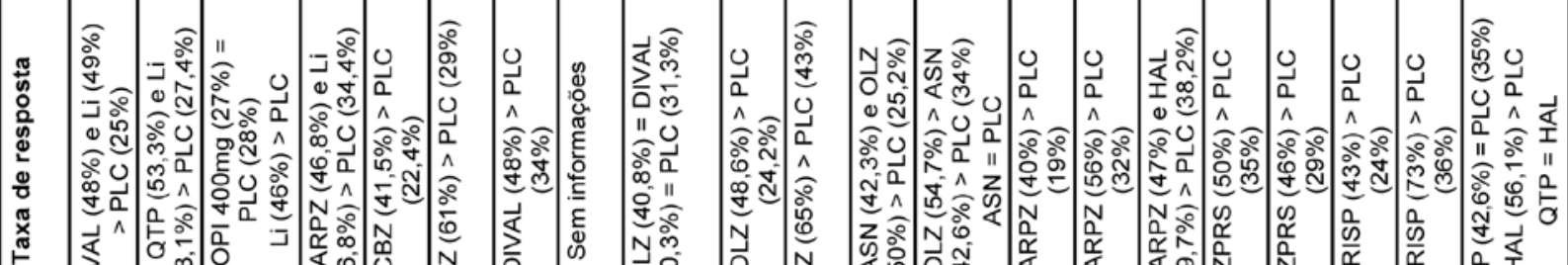

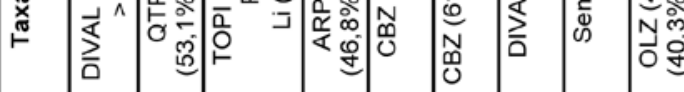

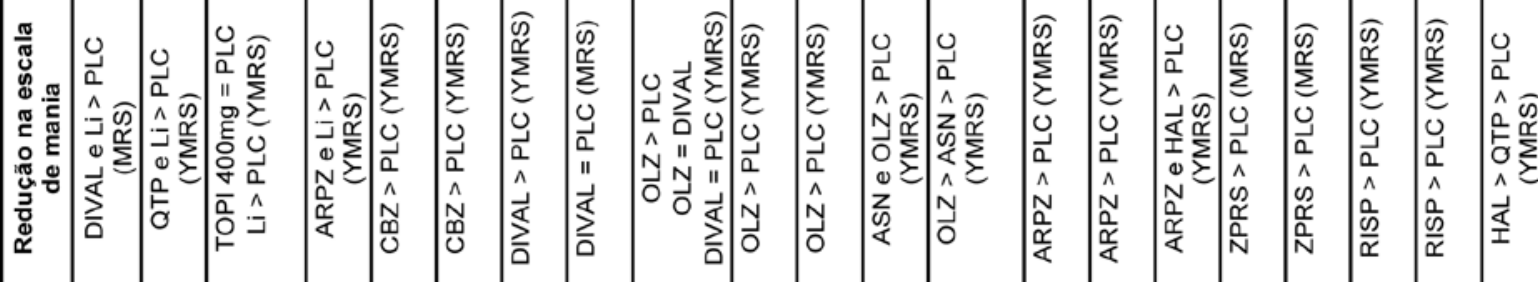

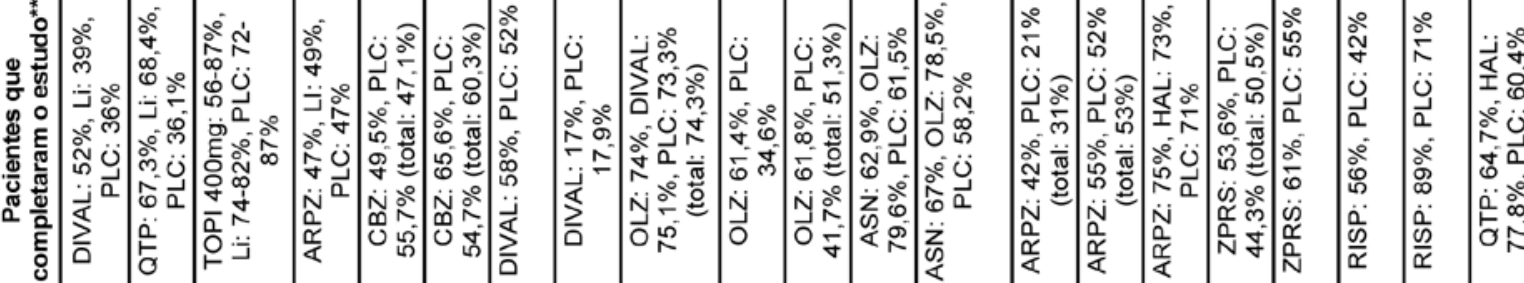

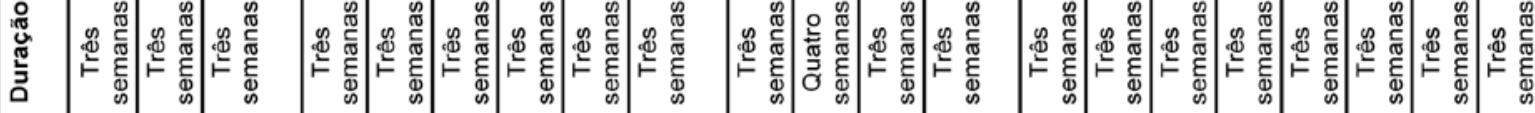

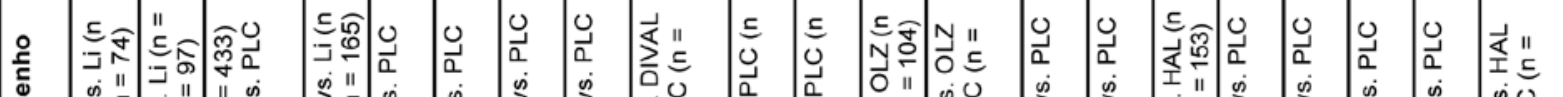

茫

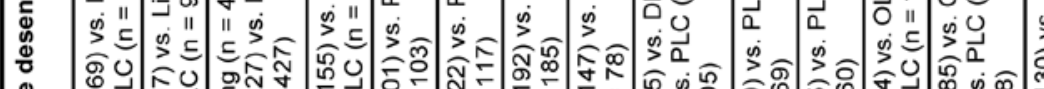

(2) "1

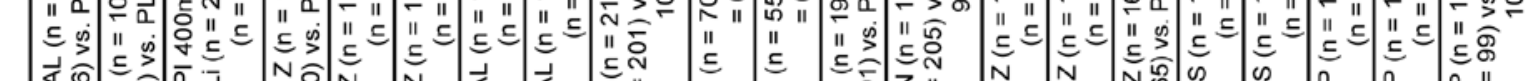

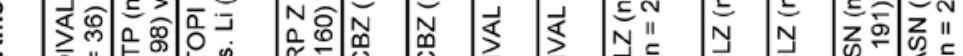

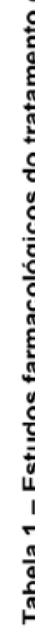

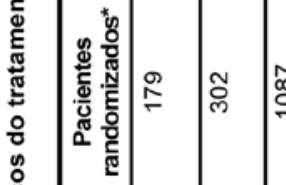

$$
\text { 添 }
$$

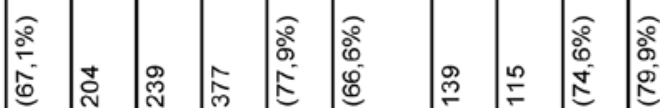
每

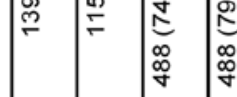

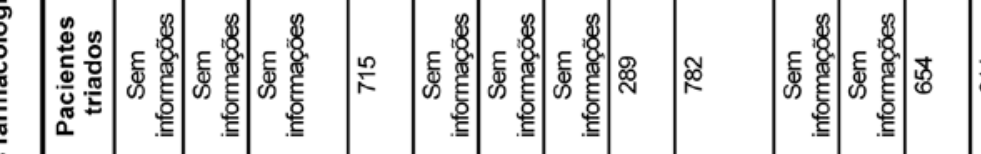

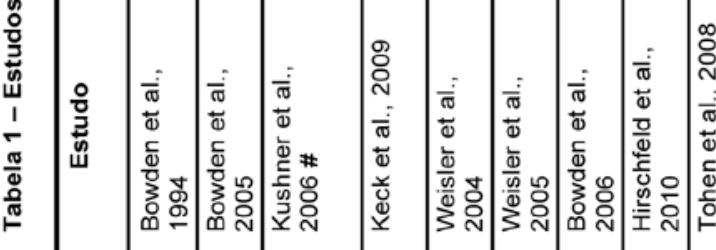

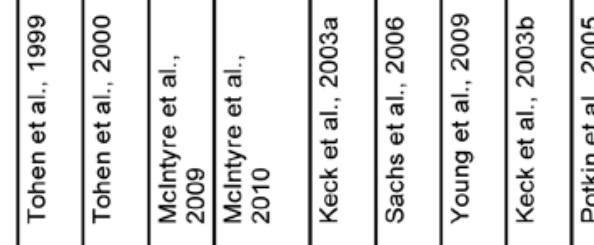

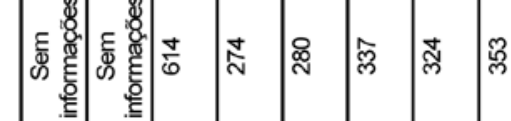
i. एं

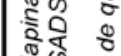
क्ष० : 这表 
em 13 não há nenhuma informação sobre o número de pacientes inicialmente triados ${ }^{7,8,11,13-15,18,19,21,22}$. Dos 11 estudos restantes, em nove mais de um quinto dos pacientes foi excluído já na triagem inicial9,12,16,17,20,23-25,27. Entre esses nove estudos, dois se caracterizaram pela exclusão de cerca de um terço dos pacientes antes da fase de randomização ${ }^{12,20}$.

Observou-se que uma parte significativa dos pacientes randomizados não completou os estudos. Em 16 dos 24 estudos, pelo menos um terço dos pacientes não chegou ao fim do período previsto de tratamento, de três ou quatro semanas $^{7-9,11-15,18,19,21,22,24,25,27}$. Em sete desses 16 estudos, a taxa de abandono foi superior a 50\% $0^{9,11-13,18,21,27}$.

Em diversos estudos, a taxa de resposta (isto é, a proporção de pacientes que tiveram uma redução de pelo menos $50 \%$ no escore total da escala de mania) foi inferior a 50\%. Isso aconteceu em três estudos com o lítio ${ }^{7,11,12}$, em um com a carbamazepina ${ }^{13}$, em três com o divalproato ${ }^{11,15,20}$, em dois com a olanzapina ${ }^{18,20}$, em três com o aripiprazol ${ }^{12,21,23}$, em dois com a asenapina ${ }^{16,17}$, em um com a ziprasidona ${ }^{25}$, em um com a risperidona ${ }^{9}$, em um com a quetiapina $^{10}$ e em um com o haloperidol ${ }^{23}$.

Em quatro estudos, embora o medicamento tenha apresentado uma redução na escala de mania significativamente maior que o placebo, ele não se distinguiu do placebo quanto à taxa de resposta. Essa situação ocorreu com a olanzapina ${ }^{20}$, a quetiapina ${ }^{10}$, a asenapina ${ }^{17}$, o aripiprazol e o haloperidol ${ }^{23}$.

\section{Tratamento da depressão bipolar}

A Tabela 2 apresenta os dados dos sete artigos referentes ao tratamento farmacológico da depressão bipolar. Esses sete artigos trazem os resultados de 11 estudos clínicos, pois um artigo apresenta os resultados de cinco estudos clínicos ${ }^{28}$, outro artigo é relativo a outros dois estudos clínicos ${ }^{29}$ e as informações de um mesmo estudo clínico são expostas em dois artigos ${ }^{28,30}$. Com uma única exceção ${ }^{31}$, os estudos clínicos compararam uma única substância ao placebo. Em todos os estudos, foi utilizada a redução no escore total de uma escala de depressão como medida primária de eficácia.

Num mesmo estudo ${ }^{31}$, a combinação olanzapina-fluoxetina e a olanzapina distinguiram-se do placebo, sendo que a combinação olanzapina-fluoxetina foi também superior à olanzapina. A quetiapina foi avaliada em três estudos ${ }^{32-34}$ e, em todos eles, foi superior ao placebo. Já os cinco estudos com a lamotrigina foram negativos, embora, em um deles, ela tenha se diferenciado do placebo numa medida secundária de eficácia ${ }^{28,30}$. Os dois estudos com o aripiprazol também foram negativos ${ }^{29}$.

Em dez dos 11 estudos, a substância investigada não se distinguiu do placebo quanto à taxa de virada para mania ou hipomania ${ }^{28-32,34}$. No estudo restante ${ }^{33}$, a quetiapina levou a um número significativamente menor de viradas que o placebo.

As amostras dos estudos sobre o tratamento da depressão bipolar foram grandes, variando de $195^{30}$ a $833^{31}$ pacientes. No entanto, muitos pacientes avaliados na triagem inicial não chegaram à etapa de randomização do estudo em função da aplicação de diversos critérios de exclusão. Entre estes critérios, foram comuns: comorbidade com outro transtorno mental do eixo I, especialmente um transtorno de abuso ou dependência de substância, risco importante de violência ou suicídio e doença clínica significativa. Em sete dos 11 estudos não há informações sobre quantos pacientes foram excluídos já na primeira etapa. Nos quatro estudos restantes, foram incluídos de $64,6 \%{ }^{33}$ a $77,7 \%{ }^{31}$ dos pacientes avaliados na triagem inicial. Com exceção de um estudo ${ }^{31}$, em todos os outros as amostras eram compostas exclusivamente por pacientes ambulatoriais.

Em oito dos 11 estudos, mais de um terço dos pacientes randomizados abandonou o tratamento antes do final do período estipulado, de sete a dez semanas ${ }^{28,29,31-34}$. Em um desses oito estudos, essa taxa foi superior a $50 \%{ }^{31}$.

Com relação às taxas de resposta, a lamotrigina ${ }^{28,30}$ e o aripiprazol $^{29}$ não se distinguiram do placebo. Embora a taxa de resposta com a olanzapina tenha sido significativamente superior à obtida com o placebo, ela foi de apenas 39\%.

\section{Tratamento de manutenção no transtorno bipolar}

A Tabela 3 apresenta os resultados dos seis artigos que investigaram o tratamento de manutenção no transtorno bipolar, que correspondem a seis estudos diferentes. Em todos os estudos, os pacientes foram randomizados após alcançarem remissão sintomática de um episódio afetivo agudo, durante o qual foram tratados de forma aberta. Em cinco estudos ${ }^{35-39}$, os pacientes tinham apresentado um episódio maníaco, hipomaníaco ou misto e, no estudo restante ${ }^{40}$, um episódio depressivo. Nos seis estudos, a medida primária de eficácia foi o tempo para a recorrência de um episódio afetivo. Dependendo do estudo, o que indicava o surgimento de um novo episódio afetivo era um aumento dos escores nas escalas de mania ou depressão, a necessidade de associar uma nova substância ao tratamento, ou hospitalização.

Num estudo com o divalproato e o lítio ${ }^{35}$, ambas as substâncias não se diferenciaram do placebo quanto à prevenção de novos episódios afetivos. Os dois estudos com a lamotrigina e o lítio apresentaram resultados idênticos: as duas substâncias foram superiores ao placebo na prevenção de episódios afetivos em geral; o lítio se distinguiu do placebo na prevenção de episódios maníacos, mas a lamotrigina não; e a lamotrigina se distinguiu do placebo na prevenção de episódios depressivos, mas o lítio não ${ }^{36,40}$. Já a olanzapina foi superior ao placebo tanto na profilaxia de episódios maníacos como de depressivos ${ }^{39}$. Por fim, os estudos com o aripiprazol ${ }^{37}$ e com a risperidona injetável de ação prolongada $^{38}$ tiveram resultados semelhantes: as duas substâncias se diferenciaram do placebo na prevenção de episódios afetivos em geral e maníacos, mas não na prevenção de episódios depressivos.

Os tamanhos das amostras nos seis estudos variaram de $161^{37} \mathrm{a}$ $463^{40}$ pacientes. Apenas uma parte dos pacientes tratados durante o episódio afetivo agudo tornou-se eutímica e pôde ser randomizada: entre $25,4 \%{ }^{37}$ e $65,1 \%{ }^{35}$. Nos dois estudos com a lamotrigina e o lítio $^{36,40}$, foram randomizados somente os pacientes que tinham apresentado remissão sintomática com o uso da lamotrigina. 


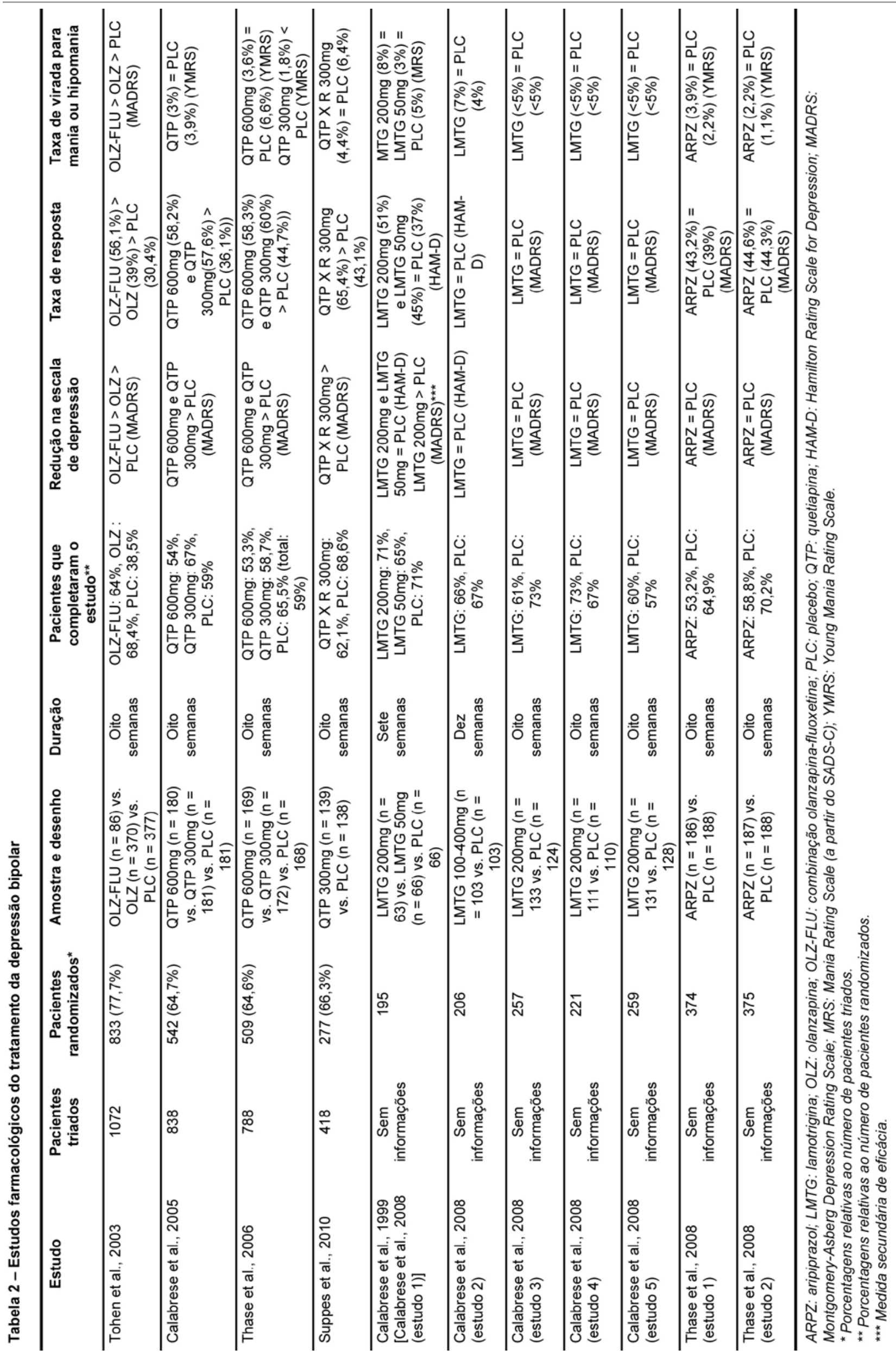




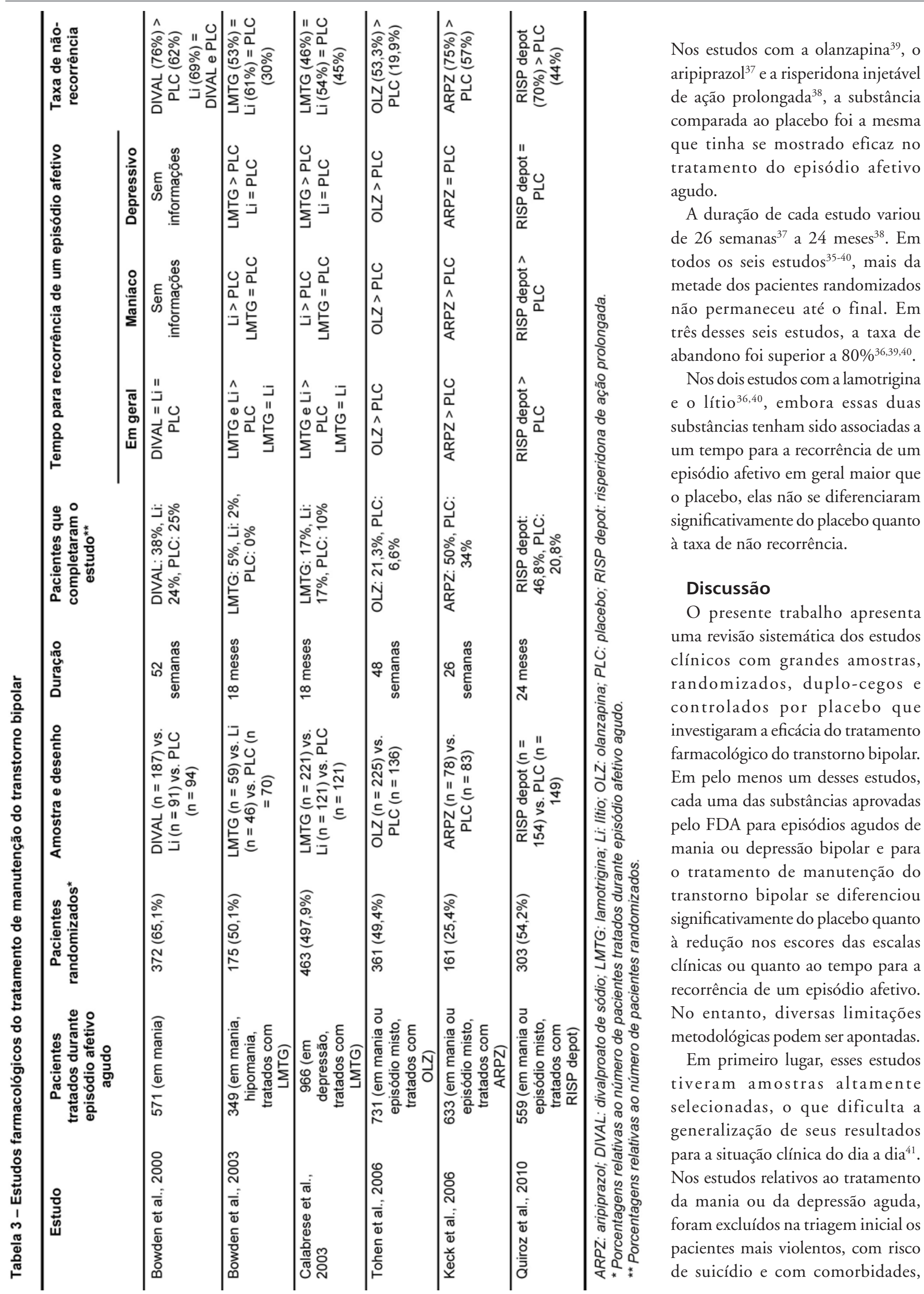


especialmente com transtornos mentais relacionados a substâncias. Ou seja, foram excluídos justamente os pacientes mais graves. Como exemplo dessa menor gravidade clínica está o fato de as amostras nos estudos sobre o tratamento da depressão bipolar, com uma única exceção ${ }^{31}$, terem sido constituídas unicamente por pacientes ambulatoriais.

Com relação a quase todos os estudos sobre o tratamento de manutenção, houve um importante viés na seleção das amostras: a substância testada após a randomização era a mesma que tinha sido eficaz no tratamento de um episódio afetivo agudo, numa etapa aberta do estudo. Assim, por exemplo, a olanzapina foi superior ao placebo na prevenção de novos episódios afetivos numa amostra de pacientes que, imediatamente antes, quando estavam em mania, já tinham apresentado uma resposta favorável à mesma olanzapina ${ }^{39}$. O mesmo pode ser dito em relação à lamotrigina ${ }^{36,40}$, ao aripiprazol ${ }^{37}$ e à risperidona injetável de ação prolongada ${ }^{38}$.

Um aspecto metodológico importante foram as altas taxas de abandono, o que enviesa os resultados do desfecho, mesmo quando se aplicam os princípios da "intenção de se tratar" e da "última observação levada adiante" na análise dos resultados ${ }^{42}$. Nos estudos sobre o tratamento da mania e da depressão agudas, cerca de $40 \%$ dos pacientes em média não concluíram o tratamento. Em relação aos estudos sobre o tratamento de manutenção, as taxas de abandono foram em média $75 \%$.

Outro elemento que merece destaque se refere às taxas de resposta, que, em vários estudos sobre o tratamento da mania, foram inferiores a $50 \%$ dos pacientes. Isso aconteceu com todas as substâncias testadas em pelo menos um estudo. Além disso, em quatro estudos - com a olanzapina ${ }^{20}$, a quetiapina ${ }^{10}$, a asenapina ${ }^{17}$, o aripiprazol e o haloperidol ${ }^{23}$ - as substâncias foram consideradas eficazes, mas não se distinguiram significativamente do placebo quanto à proporção de pacientes considerados respondedores. Com relação à depressão bipolar, apenas 39\% dos pacientes que fizeram uso de olanzapina tiveram uma resposta favorável ${ }^{31}$. Por fim, em dois estudos a lamotrigina e o lítio foram equivalentes ao placebo em relação às taxas de não recorrência de episódios afetivos no tratamento de manutenção do transtorno bipolar ${ }^{36,40}$.

$\mathrm{Na}$ análise da eficácia do tratamento farmacológico do transtorno bipolar, deve-se considerar ainda que os estudos financiados pela indústria farmacêutica que apresentam um resultado negativo frequentemente não são publicados ${ }^{43,44}$. Assim, há um importante viés se levarmos em conta apenas os estudos publicados. Por outro lado, algumas vezes acontece de o estudo negativo ser publicado, mas a interpretação dos resultados dada pelos autores ser tendenciosa ${ }^{43}$. Este foi o caso de um estudo com a lamotrigina na depressão bipolar ${ }^{30}$. Embora a substância tenha sido equivalente ao placebo na medida primária de eficácia, a escala de Hamilton para depressão, os autores enfatizaram diferenças em medidas secundárias de eficácia, como a escala de Montgomery-Asberg, e concluíram que "a lamotrigina em monoterapia é um tratamento eficaz e bem tolerado para a depressão bipolar”.

O que também dificulta uma melhor avaliação sobre as opções para o tratamento do transtorno bipolar é a escassez de estudos com uma metodologia adequada com determinadas substâncias ou classes de substâncias. Por exemplo, os estudos com a clorpromazina e outros antipsicóticos típicos na mania são muito antigos ${ }^{5}$, com exceção de dois estudos com antipsicóticos de segunda geração nos quais o haloperidol foi utilizado como um comparador ativo ${ }^{10,23}$. Nos estudos controlados por placebo com antidepressivos no tratamento da depressão bipolar, ou a substância estava associada a outro medicamento ou na amostra havia também pacientes com depressão unipolar ${ }^{45}$. Os dois estudos com o divalproato na depressão bipolar tiveram amostras muito pequenas ${ }^{46,47}$. Por fim, embora o divalproato seja apontado com uma das principais opções no tratamento de manutenção do transtorno bipolar em diversas diretrizes terapêuticas ${ }^{48}$, no único estudo controlado com placebo publicado, esse anticonvulsivante não se diferenciou do placebo ${ }^{35}$.

\section{Conclusão}

Os resultados dos modernos estudos farmacológicos sobre o tratamento do transtorno bipolar, apesar do emprego de métodos de pesquisa bastante rigorosos e sofisticados, que seguem os padrôes científicos atuais, devem ser considerados com cautela. Esses estudos apresentaram algumas importantes limitações metodológicas, tais como amostras altamente selecionadas, altas taxas de abandono e baixas taxas de resposta terapêutica.

Financiamento e conflito de interesses

\begin{tabular}{|c|c|c|c|c|c|c|c|}
\hline $\begin{array}{l}\text { Membro do grupo } \\
\text { de autores }\end{array}$ & $\begin{array}{l}\text { Local de } \\
\text { trabalho }\end{array}$ & $\begin{array}{l}\text { Verba de } \\
\text { pesquisa }^{1}\end{array}$ & $\begin{array}{c}\text { Outro apoio à } \\
\text { pesquisa ou educação } \\
\text { médica continuada }\end{array}$ & $\begin{array}{l}\text { Honorários } \\
\text { de } \\
\text { palestrantes }\end{array}$ & $\begin{array}{l}\text { Participação } \\
\text { acionária }\end{array}$ & $\begin{array}{l}\text { Consultorl } \\
\text { conselho } \\
\text { consultivo }\end{array}$ & Outro $^{3}$ \\
\hline \multicolumn{8}{|c|}{$\begin{array}{l}\text { * Modesto } \\
\text { ** Significativa } \\
\text { *** Significativa. Montantes formecidos à instituição do autor ou a colega onde o autor tem participação, não diretamente ao autor. } \\
\text { Nota: IPUB-UFRJ = Instituto de Psiquiatria da Universidade Federal do Rio de Janeiro; FCM-UERJ = Faculdade de Ciências Médicas da } \\
\text { Universidade do Estado do Rio de Janeiro. } \\
\text { Mais informações, consultar as Instruçoes aos Autores. }\end{array}$} \\
\hline
\end{tabular}




\section{Referências}

1. Fountoulakis KN, Vieta E, Siamouli M, Valenti M, Magiria S, Oral T, Fresno D, Giannakopoulos P, Kaprinis GS. Treatment of bipolar disorder: a complex treatment for a multi-faceted disorder. Ann Gen Psychiatry. 2007;6:27.

2. Tarazi FI, Shahid M. Asenapine maleate: a new drug for the treatment of schizophrenia and bipolar mania. Drugs Today (Barc). 2009;45(12):86576.

3. Azorin JM, Kaladjian A. An update on the treatment of bipolar depression. Expert Opin Pharmacother. 2009;10(2):161-72.

4. McIntyre RS, Woldeyohannes HO, Yasgur BS, Soczynska JK, Miranda A, Konarski JZ. Maintenance treatment in bipolar disorder: a focus on aripiprazole. Expert Rev Neurother. 2007;7(8):919-25.

5. McElroy SL, Keck PE Jr. Pharmacologic agents for the treatment of acute bipolar mania. Biol Psychiatry. 2000;48(6):539-57.

6. Perlis RH, Ostacher MJ, Patel JK, Marangell LB, Zhang H, Wisniewski SR, Ketter TA, Miklowitz DJ, Otto MW, Gyulai L, Reilly-Harrington N A, Nierenberg AA, Sachs GS, Thase ME. Predictors of recurrence in bipolar disorder: primary outcomes from the Systematic Treatment Enhancement Program for Bipolar Disorder (STEP-BD). Am J Psychiatry. 2006;163(2):217-24.

7. Kushner SF, Khan A, Lane R, Olson WH. Topiramate monotherapy in the management of acute mania: results of four double-blind placebocontrolled trials. Bipolar Disord. 2006;8(1):15-27.

8. Bowden CL, Grunze H, Mullen J, Brecher M, Paulsson B, Jones M, Vagero M, Svensson K. A randomized, double-blind, placebo-controlled efficacy and safety study of quetiapine or lithium as monotherapy for mania in bipolar disorder. J Clin Psychiatry. 2005;66(1):111-21.

9. Hirschfeld RM, Keck PE Jr, Kramer M, Karcher K, Canuso C, Eerdekens M, Grossman F. Rapid antimanic effect of risperidone monotherapy: a 3-week multicenter, double-blind, placebo-controlled trial. Am J Psychiatry. 2004;161(6):1057-65.

10. McIntyre RS, Brecher M, Paulsson B, Huizar K, Mullen J. Quetiapine or haloperidol as monotherapy for bipolar mania--a 12-week, doubleblind, randomised, parallel-group, placebo-controlled trial. Eur Neuropsychopharmacol. 2005;15(5):573-85.

11. Bowden CL, Brugger AM, Swann AC, Calabrese JR, Janicak PG, Petty F, Dilsaver SC, Davis JM, Rush AJ, Small JG. Efficacy of divalproex vs lithium and placebo in the treatment of mania. The Depakote Mania Study Group. JAMA. 1994;271(12):918-24.

12. Keck PE, Orsulak PJ, Cutler AJ, Sanchez R, Torbeyns A, Marcus RN, McQuade $\mathrm{RD}$, Carson WH. Aripiprazole monotherapy in the treatment of acute bipolar I mania: a randomized, double-blind, placebo- and lithiumcontrolled study. J Affect Disord. 2009;112(1-3):36-49.

13. Weisler RH, Kalali AH, Ketter TA. A multicenter, randomized, double-blind, placebo-controlled trial of extended-release carbamazepine capsules as monotherapy for bipolar disorder patients with manic or mixed episodes. J Clin Psychiatry. 2004;65(4):478-84.

14. Weisler RH, Keck PE Jr, Swann AC, Cutler AJ, Ketter TA, Kalali AH. Extendedrelease carbamazepine capsules as monotherapy for acute mania in bipolar disorder: a multicenter, randomized, double-blind, placebo-controlled trial. J Clin Psychiatry. 2005;66(3):323-30.

15. Bowden CL, Swann AC, Calabrese JR, Rubenfaer LM, Wozniak PJ, Collins MA, Abi-Saab W, Saltarelli M. A randomized, placebo-controlled, multicenter study of divalproex sodium extended release in the treatment of acute mania. J Clin Psychiatry. 2006;67(10):1501-10.

16. McIntyre RS, Cohen M, Zhao J, Alphs L, Macek TA, Panagides J. A 3-week, randomized, placebo-controlled trial of asenapine in the treatment of acute mania in bipolar mania and mixed states. Bipolar Disord. 2009;11(7):673-86.

17. McIntyre RS, Cohen M, Zhao J, Alphs L, Macek TA, Panagides J. Asenapine in the treatment of acute mania in bipolar I disorder: a randomized, doubleblind, placebo-controlled trial. J Affect Disord. 2010;122(1-2):27-38.

18. Tohen M, Sanger TM, McElroy SL, Tollefson GD, Chengappa KN, Daniel DG, Petty F, Centorrino F, Wang R, Grundy SL, Greaney MG, Jacobs TG, David SR, Toma V. Olanzapine versus placebo in the treatment of acute mania. Olanzapine HGEH Study Group. Am J Psychiatry. 1999;156(5):702-9.

19. Tohen M, Jacobs TG, Grundy SL, McElroy SL, Banov MC, Janicak PG, Sanger T, Risser R, Zhang F, Toma V, Francis J, Tollefson GD, Breier A.. Efficacy of olanzapine in acute bipolar mania: a double-blind, placebo-controlled study. The Olanzipine HGGW Study Group. Arch Gen Psychiatry. 2000;57(9):841-9.
20. Tohen M, Vieta E, Goodwin GM, Sun B, Amsterdam JD, Banov M, Shekhar A, Aaronson ST, Bardenstein L, Grecu-Gabos I, Tochilov V, Prelipceanu D, Oliff HS, Kryzhanovskaya L, Bowden C. Olanzapine versus divalproex versus placebo in the treatment of mild to moderate mania: a randomized, 12-week, double-blind study. J Clin Psychiatry. 2008;69(11):1776-89.

21. Keck PE Jr, Marcus R, Tourkodimitris S, Ali M, Liebeskind A, Saha A, Ingenito G. A placebo-controlled, double-blind study of the efficacy and safety of aripiprazole in patients with acute bipolar mania. Am J Psychiatry. 2003;160(9):1651-8.

22. Sachs G, Sanchez R, Marcus R, Stock E, McQuade R, Carson W, AbouGharbia N, Impellizzeri C, Kaplita S, Rollin L, Iwamoto T. Aripiprazole in the treatment of acute manic or mixed episodes in patients with bipolar I disorder: a 3-week placebo-controlled study. J Psychopharmacol. 2006;20(4):536-46.

23. Young AH, Oren DA, Lowy A, McQuade RD, Marcus RN, Carson WH, Spiller NH, Torbeyns AF, Sanchez R. Aripiprazole monotherapy in acute mania: 12-week randomised placebo- and haloperidol-controlled study. Br J Psychiatry. 2009;194(1):40-8.

24. Keck PE, Jr., Versiani M, Potkin S, West SA, Giller E, Ice K. Ziprasidone in the treatment of acute bipolar mania: a three-week, placebo-controlled, double-blind, randomized trial. Am J Psychiatry. 2003b;160(4):741-8.

25. Potkin SG, Keck PE, Jr., Segal S, Ice K, English P. Ziprasidone in acute bipolar mania: a 21-day randomized, double-blind, placebo-controlled replication trial. J Clin Psychopharmacol. 2005;25(4):301-10.

26. Khanna S, Vieta E, Lyons B, Grossman F, Eerdekens M, Kramer M. Risperidone in the treatment of acute mania: double-blind, placebo-controlled study. Br J Psychiatry. 2005;187:229-34.

27. Hirschfeld RM, Bowden CL, Vigna NV, Wozniak P, Collins M. A randomized, placebo-controlled, multicenter study of divalproex sodium extendedrelease in the acute treatment of mania. J Clin Psychiatry. 2010;71(4):42632.

28. Calabrese JR, Huffman RF, White RL, Edwards S, Thompson TR, Ascher JA, Monaghan ET, Leadbetter RA. Lamotrigine in the acute treatment of bipolar depression: results of five double-blind, placebo-controlled clinical trials. Bipolar Disord. 2008;10(2):323-33.

29. Thase ME, Jonas A, Khan A, Bowden CL, Wu X, McQuade RD, Carson WH, Marcus RN, Owen R. Aripiprazole monotherapy in nonpsychotic bipolar I depression: results of 2 randomized, placebo-controlled studies. J Clin Psychopharmacol. 2008;28(1):13-20.

30. Calabrese JR, Bowden CL, Sachs GS, Ascher JA, Monaghan E, Rudd GD. A double-blind placebo-controlled study of lamotrigine monotherapy in outpatients with bipolar I depression. Lamictal 602 Study Group. J Clin Psychiatry. 1999;60(2):79-88.

31. Tohen M, Vieta E, Calabrese J, Ketter TA, Sachs G, Bowden C, Mitchell P B, Centorrino F, Risser R, Baker RW, Evans AR, Beymer K, Dube S, Tollefson GD, Breier A. Efficacy of olanzapine and olanzapine-fluoxetine combination in the treatment of bipolar I depression. Arch Gen Psychiatry. 2003;60(11):1079-88

32. Calabrese JR, Keck PE Jr, Macfadden W, Minkwitz M, Ketter TA, Weisler RH, Cutler AJ, McCoy R, Wilson E, Mullen J. A randomized, double-blind, placebo-controlled trial of quetiapine in the treatment of bipolar I or II depression. Am J Psychiatry. 2005;162(7):1351-60.

33. Thase ME, Macfadden W, Weisler RH, Chang W, Paulsson B, Khan A, Calabrese JR. Efficacy of quetiapine monotherapy in bipolar I and II depression: a double-blind, placebo-controlled study (the BOLDER II study). J Clin Psychopharmacol. 2006;26(6):600-9.

34. Suppes T, Datto C, Minkwitz M, Nordenhem A, Walker C, Darko D. Effectiveness of the extended release formulation of quetiapine as monotherapy for the treatment of acute bipolar depression. J Affect Disord. 2010;121(1-2):106-15.

35. Bowden CL, Calabrese JR, McElroy SL, Gyulai L, Wassef A, Petty F, Pope HG, Chou JC, Keck PE, Rhodes LJ, Swann AC, Hirschfeld RM, Wozniak PJ. A randomized, placebo-controlled 12-month trial of divalproex and lithium in treatment of outpatients with bipolar I disorder. Divalproex Maintenance Study Group. Arch Gen Psychiatry. 2000;57(5):481-9.

36. Bowden CL, Calabrese JR, Sachs G, Yatham LN, Asghar SA, Hompland M, Montgomery P, Earl N, Smoot TM, DeVeaugh-Geiss J. A placebocontrolled 18-month trial of lamotrigine and lithium maintenance treatment in recently manic or hypomanic patients with bipolar I disorder. Arch Gen Psychiatry. 2003;60(4):392-400.

37. Keck PE Jr, Calabrese JR, McQuade RD, Carson WH, Carlson BX, Rollin LM, Marcus RN, Sanchez R. A randomized, double-blind, placebo-controlled 
26-week trial of aripiprazole in recently manic patients with bipolar I disorder. J Clin Psychiatry. 2006;67(4):626-37.

38. Quiroz JA, Yatham LN, Palumbo JM, Karcher K, Kushner S, Kusumakar V. Risperidone long-acting injectable monotherapy in the maintenance treatment of bipolar I disorder. Biol Psychiatry. 2010;11(10):1727-40.

39. Tohen M, Calabrese JR, Sachs GS, Banov MD, Detke HC, Risser R, Baker RW, Chou JC, Bowden CL. Randomized, placebo-controlled trial of olanzapine as maintenance therapy in patients with bipolar I disorder responding to acute treatment with olanzapine. Am J Psychiatry. 2006; 163(2):247-56.

40. Calabrese JR, Bowden CL, Sachs G, Yatham LN, Behnke K, Mehtonen OP, Montgomery P, Ascher J, Paska W, Earl N, DeVeaugh-Geiss J. A placebocontrolled 18-month trial of lamotrigine and lithium maintenance treatment in recently depressed patients with bipolar I disorder. J Clin Psychiatry. 2003;64(9):1013-24.

41. Licht RW, Gouliaev G, Vestergaard P, Frydenberg M. Generalisability of results from randomised drug trials. A trial on antimanic treatment. $\mathrm{Br}$ J Psychiatry. 1997;170:264-7.

42. Lavori PW. Clinical trials in psychiatry: should protocol deviation censor patient data? Neuropsychopharmacology. 1992;6(1):39-48.

43. Ghaemi SN, Shirzadi AA, Filkowski M. Publication bias and the pharmaceutical industry: the case of lamotrigine in bipolar disorder. Medscape J Med. 2008;10(9):211.

44. Gorenstein C. Who pays for the impact? Considerations on conflicts of interest. Rev Bras Psiquiatr. 2003;25(3):129-30.

45. Gijsman HJ, Geddes JR, Rendell JM, Nolen WA, Goodwin GM. Antidepressants for bipolar depression: a systematic review of randomized, controlled trials. Am J Psychiatry. 2004;161(9):1537-47.

46. Davis LL, Bartolucci A, Petty F. Divalproex in the treatment of bipolar depression: a placebo-controlled study. J Affect Disord. 2005;85(3):25966.

47. Ghaemi SN, Gilmer WS, Goldberg JF, Zablotsky B, Kemp DE, Kelley ME, Bauer AD, Fleck J, Filkowski MM, Stan VA, Dunn RT. Divalproex in the treatment of acute bipolar depression: a preliminary doubleblind, randomized, placebo-controlled pilot study. J Clin Psychiatry. 2007;68(12):1840-4.

48. Fountoulakis KN, Vieta E. Treatment of bipolar disorder: a systematic review of available data and clinical perspectives. Int J Neuropsychopharmacol. 2008;11(7):999-1029. 